\title{
Hysteretic Photochromic Switching (HPS) in Doubly Doped GaN(Mg):Eu-A Summary of Recent Results
}

\author{
Paul R. Edwards ${ }^{1}\left(\mathbb{D}\right.$, Kevin P. O'Donnell ${ }^{1}{ }^{*}$, Akhilesh K. Singh ${ }^{1,2}$, Douglas Cameron ${ }^{1}$, \\ Katharina Lorenz ${ }^{3}$, Mitsuo Yamaga ${ }^{4}$, Jacob H. Leach ${ }^{5}$, Menno J. Kappers ${ }^{6}$ and \\ Michal Boćkowski ${ }^{7}$ \\ 1 SUPA Department of Physics, University of Strathclyde, 107 Rottenrow, Glasgow G4 0NG, UK; \\ paul.edwards@strath.ac.uk (P.R.E.); akhilesh_singh343@yahoo.com (A.K.S.); \\ douglas.cameron.2013@uni.strath.ac.uk (D.C.) \\ 2 Alternative Energy Materials, CSIR-National Physical Laboratory, New Delhi-110012, India \\ 3 INESC/MN, IPFN, Instituto Superior Técnico, Universidade de Lisboa, Campus Tecnológico e Nuclear, \\ Estrada Nacional 10, 2695-066 Bobadela LRS, Portugal; lorenz@ctn.tecnico.ulisboa.pt \\ 4 Department of Mathematical and Design Engineering, Gifu University, Gifu 501-1193, Japan; \\ yamaga@gifu-u.ac.jp \\ 5 Kyma Technologies, 8829 Midway West Rd, Raleigh, NC 27612, USA; leach@kymatech.com \\ 6 Department of Materials Science and Metallurgy, University of Cambridge, 27 Charles Babbage Road, \\ Cambridge CB3 0FS, UK; mjk30@cam.ac.uk \\ 7 Institute of High Pressure Physics PAS, Sokolowska 29/37, 01-142 Warsaw, Poland; bocian@unipress.waw.pl \\ * Correspondence: k.p.odonnell@strath.ac.uk; Tel.: +44-141-548-3365
}

Received: 6 July 2018; Accepted: 19 September 2018; Published: 22 September 2018

check for updates

\begin{abstract}
Europium is the most-studied and least-well-understood rare earth ion (REI) dopant in GaN. While attempting to increase the efficiency of red GaN light-emitting diodes (LEDs) by implanting $\mathrm{Eu}^{+}$into p-type $\mathrm{GaN}$ templates, the Strathclyde University group, in collaboration with IST Lisbon and Unipress Warsaw, discovered hysteretic photochromic switching (HPS) in the photoluminescence spectrum of doubly doped $\mathrm{GaN}(\mathrm{Mg})$ :Eu. Our recent work, summarised in this contribution, has used time-, temperature- and light-induced changes in the Eu intra- $4 f$ shell emission spectrum to deduce the microscopic nature of the $\mathrm{Mg}$-Eu defects that form in this material. As well as shedding light on the $\mathrm{Mg}$ acceptor in $\mathrm{GaN}$, we propose a possible role for these emission centres in quantum information and computing.
\end{abstract}

Keywords: gallium nitride; rare earth ions; europium; photoluminescence; photochromism; qubit

\section{Introduction}

The doping of III-nitride semiconductors with rare earth ions, largely motivated by the promise of high-efficiency red GaN-based LEDs, has a fairly recent history [1]. By contrast, the $p$-type activation of $\mathrm{GaN}$ is well established, and underpins all commercial activities associated with the blue LED, although the nature of the $\mathrm{Mg}$ acceptor remains controversial.

In recent experiments, first described in [2], we implanted Eu as a 'spectator ion' to probe the lattice location of $\mathrm{Mg}$ in $\mathrm{GaN}(\mathrm{Mg}): \mathrm{Eu}$ [3]. We observed photochromic switching between different configurations of an Eu-Mg defect. By first cooling the sample in the dark and then using photoluminescence to monitor the process at a range of fixed temperatures, excitation densities and wavelengths, we managed to tune the characteristic switching time over many orders of magnitude. Linking the distinct Eu-Mg defect configurations with the shallow transient and deep ground states (STS and DGS) of the Mg acceptor, in the Lany-Zunger (L-Z) model [4], we determined an energy barrier of $27.7 \mathrm{meV}$ for transitions between STS and DGS in agreement with theory. The experimental 
results suggest that at low temperatures holes are localized in deep ground states on $\mathrm{N}$ atoms axially bonded to $\mathrm{Mg}$, as predicted by Lyons et al. [5].

The unexpected observation of transitions from excited ${ }^{5} \mathrm{D}_{1}$ levels of Eu at lower temperatures completes our spectroscopic survey and explains an apparent anomaly in the temperature dependences of ${ }^{5} \mathrm{D}_{0} \rightarrow{ }^{7} \mathrm{~F}_{J}$ transitions [6]. Prolonged exposure of state-of the-art Eu-implanted HVPE GaN leads to photodissociation of the Eu-Mg centres when migrating $\mathrm{Mg}$ atoms fail to return, eventually leading to emergence of emission from un-associated Eu2 defects [7]. The spectroscopic effects of triple doping $\mathrm{GaN}(\mathrm{Mg})$ :Eu with $\mathrm{O}$ or $\mathrm{N}$ are briefly described.

\section{Materials and Methods}

In the GaN lattice, Eu substitutes for Ga and takes the charge state of 3+ with 6 electrons in the $4 f$ shell. Hund's rules predict a multi-electron ground state, labelled ${ }^{7} \mathrm{~F}_{0}$ in Russells-Saunders atomic term notation $\left({ }^{2 S+1} \mathrm{~L}_{\mathrm{J}}\right)$, with spin and orbital components of $3 \hbar$ opposed to produce a total angular momentum $J=0$. The states ${ }^{7} \mathrm{~F}_{0,1,2,3}$ form a close-lying ground state manifold connected to the ${ }^{5} \mathrm{D}_{0,1,2}$ excited states by optical transitions of various types. (States lying higher than ${ }^{5} \mathrm{D}_{1}$ are not considered here.) The atomic-like states are split further in the symmetric environment of the GaN crystal field, providing a valuable clue to the actual defect configurations, which may be reduced from the expected quasi- $C_{3 v}$ symmetry of a substitutional site by the presence of intrinsic or extrinsic defects, resulting in what is called 'site multiplicity' [8]. For example, singlet levels with $J=0$ do not split in any crystal field, while the $J=1$ triplets split into doublet and singlet in an axial crystal field and into three separate levels in a non-axial field [9].

Different sites can sometimes be distinguished also by selective excitation [10,11]. For example, Eu2 defects, consisting of unassociated Eu atoms on Ga sites [11], can be excited only by light with photon energy greater than the GaN bandgap; Eu1 defects, associated with a vacancy [12], can be excited in addition by a broad band of photon energies just below the gap [10].

Doubly doping $\mathrm{GaN}$ to produce $\mathrm{GaN}(\mathrm{Mg})$ :Eu samples proceeds in two stages. GaN doped with $\sim 10^{18}$ to $10^{20} \mathrm{~cm}^{-3} \mathrm{Mg}$ is grown at Cambridge by metal organic vapour phase epitaxy (MOVPE) as a $300 \mathrm{~nm}$ layer on an undoped $3 \mu \mathrm{m} \mathrm{GaN}$ template on $50 \mathrm{~mm}$ sapphire wafers. $1 \times 1 \mathrm{~cm}^{2}$ offcuts are implanted by Eu ions in Lisbon to produce an approximately uniform depth profile, as shown in Figure 1. The lattice damage that results from ion implantation is annealed out under high pressure at high temperature in Warsaw $\left(1400{ }^{\circ} \mathrm{C}, 1 \mathrm{GPa}\right.$ of $\left.\mathrm{N}_{2}\right)$. Details of the sample preparation can be found in Ref. [13]. One sample was grown and Mg-doped by HVPE prior to implantation/annealing.

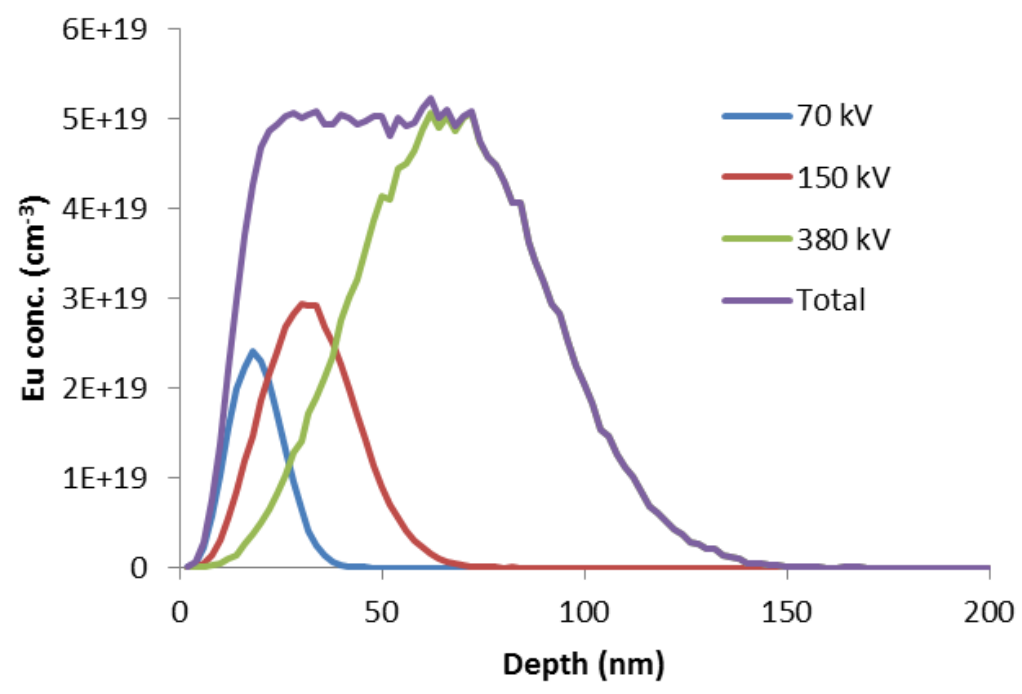

Figure 1. Ion implantation using multiple beam energies produces a top-hat dopant profile as shown in the above example, determined by Monte Carlo simulations using the SRIM code for Eu ions in GaN. 
Photoluminescence/excitation (PL/E) spectroscopy at Strathclyde is the main analytical technique used to characterize samples and examine HPS. Samples cooled in a helium cryorefrigerator are excited by monochromated light from a $1 \mathrm{~kW}$ Xe arc lamp, a $20 \mathrm{~mW} 355 \mathrm{~nm}$ laser or a pulsed nitrogen laser, in order of increasing achievable excitation density, analysed by a $2 / 3 \mathrm{~m}$ monochromator with a $1800 \mathrm{~L} / \mathrm{mm}$ holographic grating and detected with a 16 bit $1064 \times 128$ pixel CCD camera.

\section{Results}

This section will be divided into the following subheadings. In Section 3.1, we present the spectrum of as-received $\mathrm{GaN}(\mathrm{Mg})$ :Eu samples at room temperature, featuring mainly Eu0 luminescence, and introduce the 1-3-5 rule for $\mathrm{Eu}^{3+}$ defects in any host; in Section 3.2, we describe Hysteretic Photochromic Switching, as revealed in the temperature dependence of PL during a cooling-warming cycle; in Section 3.3, we describe the determination of the energy barrier that separates the photochromic defect configurations and link these observations to the Lany-Zunger model of the $\mathrm{Mg}$ acceptor; subsection Section 3.4 considers transitions from excited ${ }^{5} \mathrm{D}_{1}$ states; Section 3.5 reveals the effects of irreversible photo-dissociation of Eu-Mg defects in GaN material of exceptional quality.

\subsection{Eu0 Spectrum}

At room temperature, the typical Eu0 PL spectrum clearly exemplifies the 1-3-5 rule. Transitions from the ${ }^{5} \mathrm{D}_{0}$ excited state are identified in Figure 2 by the label of the terminal state, with degeneracies of $1\left({ }^{7} \mathrm{~F}_{0}\right), 3\left({ }^{7} \mathrm{~F}_{1}\right)$ and $5\left({ }^{7} \mathrm{~F}_{2}\right)$, respectively. All degeneracies are lifted by a crystal field with lower than axial symmetry, to produce a set of well separated lines indicated by arrows.

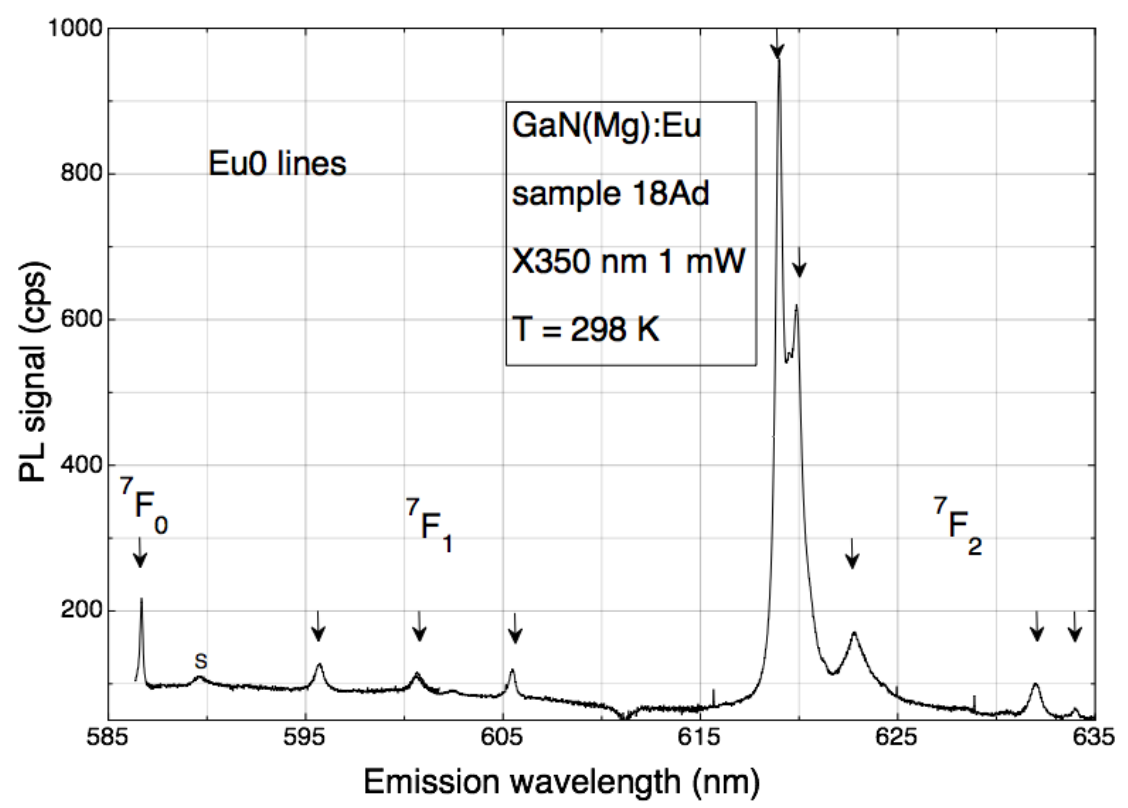

Figure 2. When excited with above-bandgap light at RT, spectrally pure GaN(Mg):Eu samples show only the Eu0 spectrum (reproduced from Ref. [9]).

The Eu0 spectrum appears only in Eu- and Mg- (double) doped GaN [1,2] and careful doping comparisons show that its maximum intensity is achieved when the concentrations of Eu and $\mathrm{Mg}$ are approximately equal. This leads us to a model of the Eu0 defect comprising a single Eu atom in close association with a Mg atom, both substituting on the Ga sub-lattice.

\subsection{HPS}

When samples are cooled below about $50 \mathrm{~K}$, we encounter (with much surprise) the photochromic switching of the Eu0 spectrum, in all of its components, to a completely different one. 
We assigned the low-temperature spectrum to $\mathrm{Eu}(\mathrm{Mg})$, due to its superficial spectral similarities with the Eu1 centre observed in undoped GaN:Eu. Eu1(Mg) is much more symmetrical than Eu0, with, most notably, the ${ }^{7} \mathrm{~F}_{1}$ state splitting as $3=2+1$, that is to say into a (close-lying) doublet and a singlet [8]. The profound alteration of the spectrum between $100 \mathrm{~K}$ and $25 \mathrm{~K}$ is shown in Figure 3.

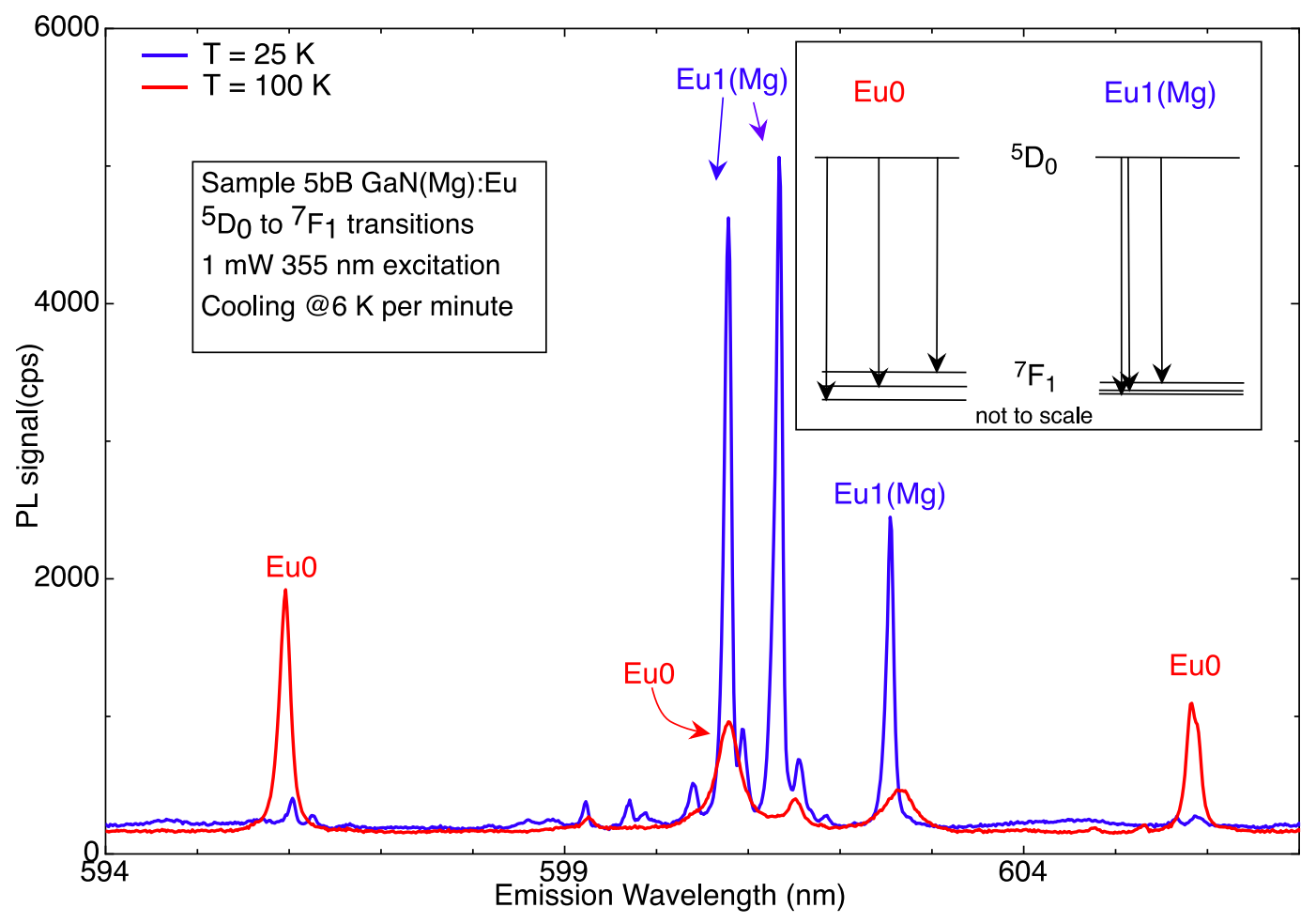

Figure 3. Showing the two very different ${ }^{5} \mathrm{D}_{0}$ to ${ }^{7} \mathrm{~F}_{1}$ spectral patterns for the photochromic pair Eu0 and $\mathrm{Eu} 1(\mathrm{Mg})$, dominant at $100 \mathrm{~K}$ and $25 \mathrm{~K}$ respectively (reproduced from Ref. [9]).

The switching of Eu0 to Eu1(Mg) is soon found to be hysteretic (hence HPS): the reverse process occurs at a much higher temperature. The different hysteretic behaviours of Eu0 and Eu1(Mg) are shown in Figure 4 for a typical experimental cycle.

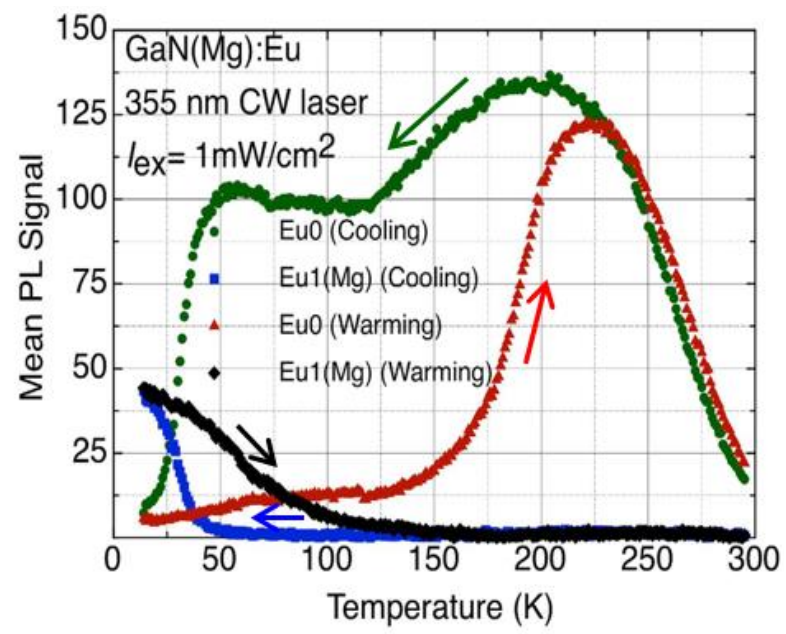

Figure 4. Showing various hysteresis loops associated with the HPS of Eu0-Eu1(Mg) during a cooling-warming cycle. The mean signal intensities are plotted against temperature for cooling and warming runs, as indicated by arrows, undertaken at a fixed rate of $6 \mathrm{~K}$ per minute. Data are recorded at $1 \mathrm{~K}$ intervals (reproduced from Ref. [3]). 
Switching upon cooling (switchdown) offers the clearest perspective: the intensity of Eu0 drops to zero over a small temperature range, while that of $\mathrm{Eu} 1(\mathrm{Mg})$ increases to a maximum value at the base temperature of our cryostat, $\sim 10 \mathrm{~K}$.

Switchback from Eu1(Mg) to Eu0 upon sample warming is more complicated and not fully understood. The maximum Eu1(Mg) signal at $10 \mathrm{~K}$ does not equal the maximum Eu0, but we ascribe this difference to one of transition probability between the more symmetric centre (less probable for intra- $f$ shell transitions) and the less symmetric one. We conclude that the switching is one-to-one with respect to defect numbers. The anomalous decrease in intensity of the Eu0 signal between its peak at $200 \mathrm{~K}$ and the start of the plateau at $125 \mathrm{~K}$, explained only recently [6], will feature in Section 3.4.

\subsection{Quantifying Photochromism}

Without a mathematical model of the temperature dependence, it is difficult to extract physical parameters from the experiments described in Section 3.2. With the realisation that the observation of photochromism requires light both to observe the effect, through PL, and to cause it, we devised a measurement protocol in which the sample was cooled to a fixed temperature in the dark and then subjected to a fixed wavelength and intensity of light for periods long enough that switching could be called essentially complete, while measuring the spectrum at 0.1 or $0.25 \mathrm{~s}$ intervals. (For brevity-and to reflect the situation in which the Mg-Eu complex is 'surprised' out of its non-equilibrium state by the sudden illumination-we familiarly refer to this as the 'Ninja!' protocol.)

Some general results can be cited for these experiments. For fixed excitation power and wavelength, the switching profile is hyperbolic in time, with a characteristic time constant $\tau$ that can be extracted from the temporal dependence of switching by fitting:

$$
I(t) / I(0)=1 /(1+t / \tau)
$$

to the dataset, where $I(t)$ is the (mean) PL intensity of the unswitched (Eu0) component at time $t$. The switching rate slows with increasing temperature, in an anti-Arrhenius relationship that reflects the fact that colder samples are further from equilibrium; it is the return to equilibrium that is captured in the Ninja! runs. Increasing the incident power decreases the switching time, as might be expected.

Some example results for a particular sample are illustrated in Figure 5a,b. By fitting the temperature dependence of the switching time, the barrier between the photochromic states is estimated to be $27.7 \mathrm{meV}$, more or less independent of the excitation power density.

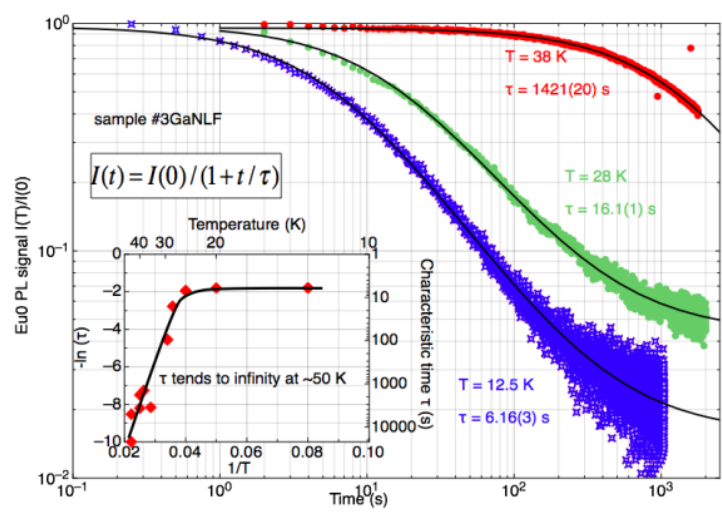

(a)

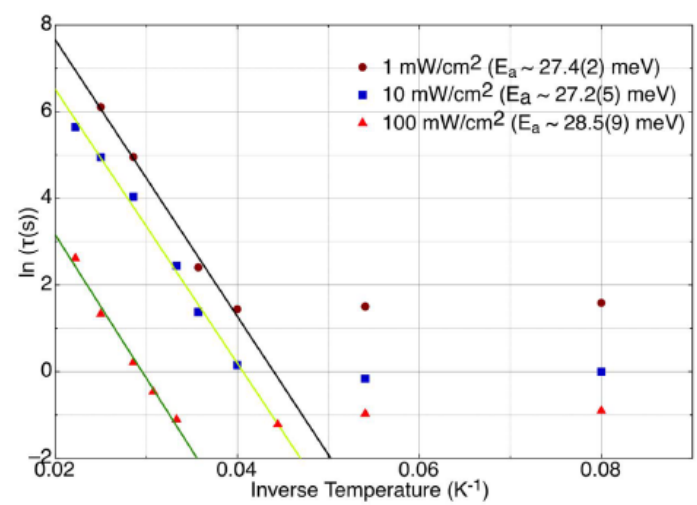

(b)

Figure 5. (a) Temperature dependence of switching at fixed excitation density and wavelength, the inset showing temperature independence below $20 \mathrm{~K}$; (b) anti-Arrhenius fits to the temperature dependence above $\sim 20 \mathrm{~K}$ show an independence of the activation energy on the power density (after Ref. [3]). 


\subsection{Transitions from ${ }^{5} D_{1}$ Levels}

In conventional PL of semiconductors, it is expected that thermalisation between excited states should decrease the relative intensity of transitions originating on states lying higher in energy as the temperature decreases. The intensity should mirror a decreasing population of the higher lying levels through the operation of Boltzmann statistics.

On the contrary, transitions from the ${ }^{5} \mathrm{D}_{1}$ levels in $\mathrm{GaN}(\mathrm{Mg})$ :Eu are stronger at lower temperatures. Such transitions for both configurations of Eu-Mg defects are shown in Figure 6, and their temperature dependences in Figure 7.
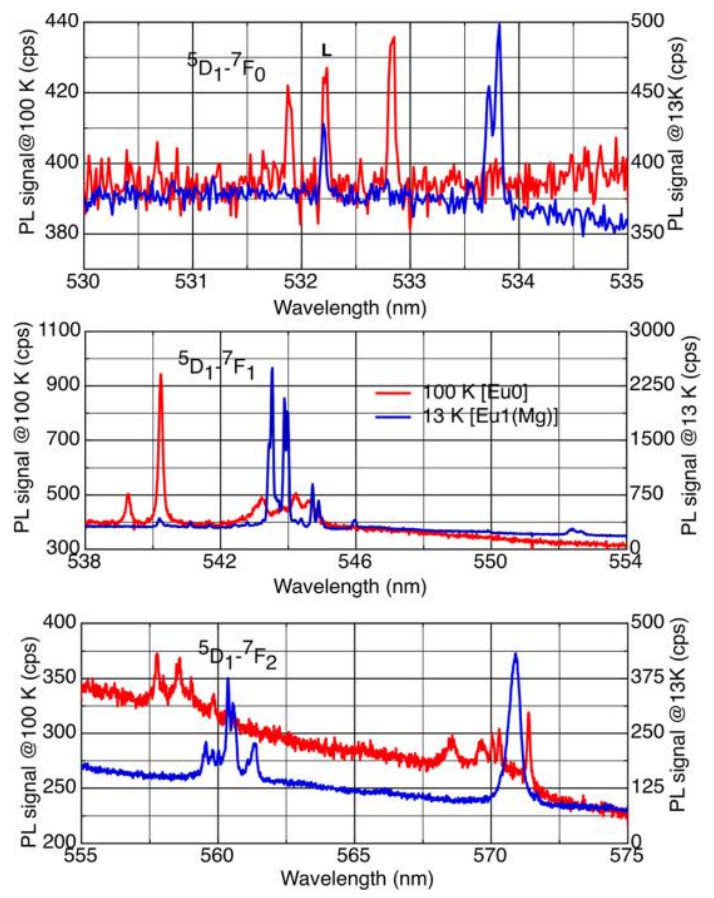

Figure 6. The transitions from the ${ }^{5} \mathrm{D}_{1}$ states of both $\mathrm{Eu} 0$ and $\mathrm{Eu} 1(\mathrm{Mg})$ configurations of the Eu-Mg defects can be observed at the appropriate temperatures, allowing for photochromic switching (reproduced from Ref. [6]). For an explanation, see text.

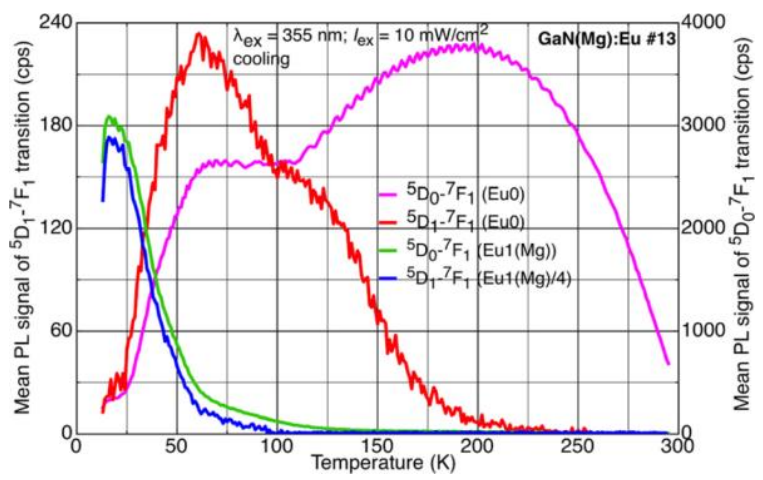

Figure 7. Temperature dependences of intensities of transitions as indicated (cooling run). Note the anti-correlation of Eu0 PL signals from the ${ }^{5} \mathrm{D}_{1}$ and ${ }^{5} \mathrm{D}_{0}$ states in the temperature range $200 \mathrm{~K}$ to $100 \mathrm{~K}$ (reproduced from Ref. [6]).

The ${ }^{7} \mathrm{~F}_{0,1,2}$ level splittings derived from an analysis of the spectra of Figure 6 confirm those found previously for the stronger transitions from the ${ }^{5} \mathrm{D}_{0}$ states to these levels. This allows us to determine the ${ }^{5} D_{1}$ to ${ }^{5} D_{0}$ energy separation as well as the (relatively small) splittings within the ${ }^{5} D_{1}$ levels caused by the crystal field [6]. 


\subsection{Photodissociation}

When samples are subjected to prolonged exposure to above-bandgap light at low temperatures, further spectral changes occur, which we refer to as 'second switching' or 'blitching': bleaching of the total emission is accompanied by switching from $\mathrm{Eu} 1(\mathrm{Mg})$ to a set of ever more symmetrical centres, labelled Eu1(Mg2), Eu1(Mg3) etc. [14-16].

In MOVPE-grown samples, second switching occurs at a leisurely pace, necessitating experimental runs as long as 36,000 s to establish clear trends. However, for a state-of-the-art free-standing $\mathrm{GaN}(\mathrm{Mg})$ sample prepared by HVPE at Kyma Technologies, and doped with Eu as described in Section 2, the blitching process is rapid, and ultimately destructive [7].

Figure 8 shows the eventual result of such an experiment. Following a first set of runs that resulted in irreversible spectral alterations, the sample was re-annealed at high temperature and pressure, but only partly recovered its original spectral purity. (A sample that has lost the Eu0 dominance of the RT spectrum, shown in Figure 2, is said to be 'cooked'.)

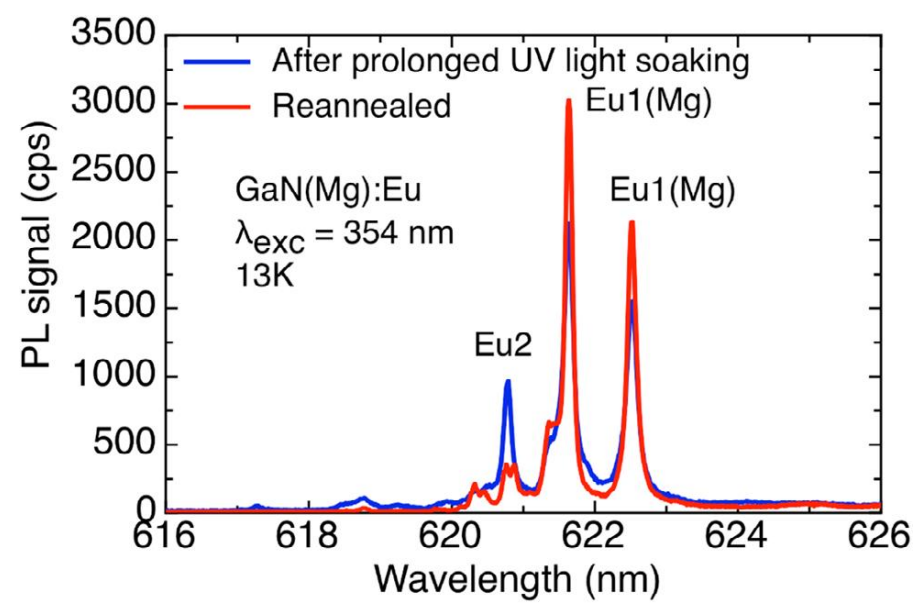

Figure 8. After prolonged exposure to UV light at low temperature, this 'half-cooked' sample displays the characteristic $620.9 \mathrm{~nm}$ line of the prime Eu2 centre, produced by unassociated substitutional $\mathrm{Eu}^{3+}$ ions [11].

\section{Discussion}

When taken together, the various pieces of experimental evidence summarised in Section 3 lead us to form a precise microscopic model of the $\mathrm{Mg}$-Eu complexes that form in p-type GaN.

We know from observing maximum Eu0 emission when $\mathrm{Mg}$ and Eu concentrations match that the centre is likely to involve a single Eu atom in close association with a single $\mathrm{Mg}$ atom. Both $\mathrm{Mg}^{2+}$ and $\mathrm{Eu}^{3+}$ will favour cationic sites in the GaN lattice, so we propose that they both substitute for $\mathrm{Ga}$ and share a common nitrogen nearest neighbour.

We next consider the change in symmetry during the switch from Eu0 to Eu1(Mg) on cooling the sample (switchdown). This indicates that a local distortion in the lattice is induced during cooling, a kind of phase change occurring on the nanoscale, and shows the $\mathrm{Eu}^{3+}$ ion to be in a higher symmetry environment in the $\mathrm{Eu} 1(\mathrm{Mg})$ state.

A further clue regarding the nature of this distortion comes from the activation energy obtained from quantifying the light-induced switching of Eu0 to Eu1(Mg) at low T: this value (27.7 meV) is close to the barrier height predicted to exist between the two $\mathrm{Mg}$ acceptor states proposed by Lany and Zunger as the 'curious case of the shallow $\mathrm{Mg}_{\mathrm{Ga}}$ deep state' [4]. Hybrid density functional calculations predicted formation of a shallow transient state, effective in the p-type activation, and a deeper ground state; further calculations by Lyons et al. [5] supported the existence of such a deep state, specifically attributing it to localization of a hole at an axial $\mathrm{N}$ atom. 
We proposed [9] that the switching between $\mathrm{Eu} 0$ and $\mathrm{Eu} 1(\mathrm{Mg})$ is the spectroscopic signature of the transition between these two Mg acceptor states, as observed by the nearby Eu 'spectator' atom. Localization of a hole on the magnesium's axial $\mathrm{N}$ bond (i.e., the deep ground state) is the stable configuration at low temperature, giving Eu1(Mg); delocalization of the hole at higher temperatures, and the resultant local lattice distortion, results in a change in the crystal field splitting and the switch to Eu0.

Our microscopic model is sketched in Figure 9.

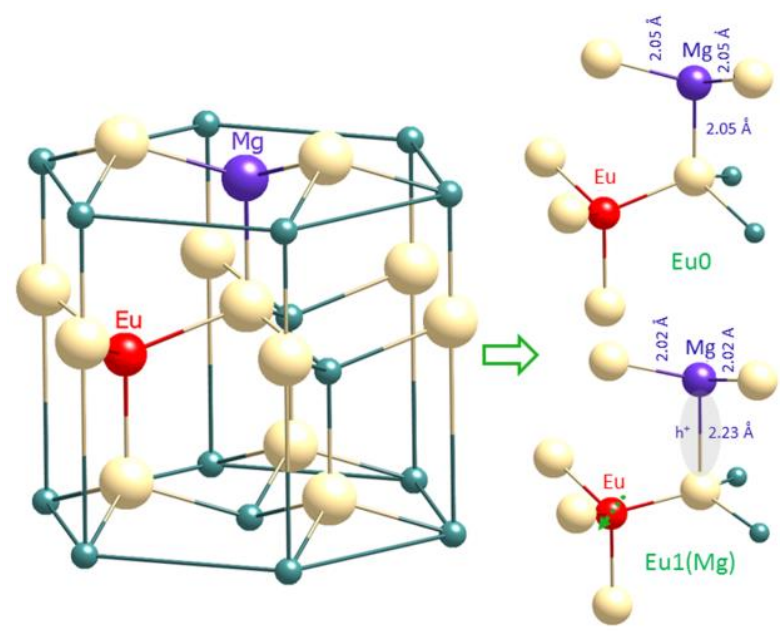

Figure 9. Proposed microscopic model showing Eu sharing a nitrogen atom axially bonded to the $\mathrm{Mg}$. The distortion of this arrangement due to the localization of a hole at this bond is also depicted (reproduced from Ref. [3]).

One effect of the prolonged UV exposure of these materials is the emergence of additional centres whose line splittings reveal successively increased symmetry [15]. This indicates that a fraction of $\mathrm{Mg}$ atoms has physically migrated to lattice sites away from their Eu partner. This eventually leads to the appearance of the Eu2 centre, identified as the 'prime' defect in unimplanted GaN:Eu (i.e., a simple substitutional $\mathrm{Eu}$ atom on a cation site); the $\mathrm{Mg}$ and $\mathrm{Eu}$ must now be sufficiently separated to be entirely disassociated.

Finally, we have made recent observations (to be fully reported at a later date) of the effects of co-implanting the $\mathrm{GaN}: \mathrm{Mg}(\mathrm{Eu})$ with additional anion species to give triply-doped material. We find that the introduction of excess $\mathrm{N}$ atoms enhances $\mathrm{Eu} 0$ production and reduces the intensity of vestigial Eu1 lines, supporting Mitchell et al.'s previous identification of this centre as substitutional Eu associated with a nitrogen vacancy [12]. The introduction of $\mathrm{O}$ results in a new centre, with the same symmetry as Eu0, but not exhibiting the switching behaviour. We interpret this as being due to the formation of an Eu-O-Mg defect complex in which the strong $\mathrm{Mg}-\mathrm{O}$ bond disrupts the ability of a hole to localise in the deep ground state which is central to the Eu0-Eu1(Mg) switching.

\section{Conclusions}

The spectroscopy of europium ions in GaN shows behaviour which is complex due to site multiplicity - and made dramatically more so by the presence of $\mathrm{Mg}$. The variation of the spectra with factors such as time, temperature, illumination and co-doping allow us to probe the mean local environment of an ensemble of $\mathrm{Eu}^{3+}$ ions, and to use these as 'spectators' to directly observe previously theorised acceptor states of the commercially important Mg dopant in GaN. Continuing work will aim to extend this to observing individual $\mathrm{Eu}^{3+}$ ions in microscope-based versions of the experiments reported in Section 3. This work will allow us to investigate the possibility of applications of REI-implanted semiconductors that go beyond the LED. We have identified in this defect a 2-level system which can be switched and read optically; learning to manipulate these states with more precise 
control has the potential to open up a new branch of solotronics (we propose the name 'QuantREIonics') aimed at quantum information and computing.

Author Contributions: Conceptualization, K.P.O.D.; Investigation, all authors; Writing: Original Draft Preparation, K.P.O.D., P.R.E.; Writing: Review and Editing, all authors; Supervision, K.P.O.D.; Project Administration, K.P.O.D., P.R.E.; Funding Acquisition, K.P.O.D., P.R.E.

Funding: This research was funded by the Engineering and Physical Sciences Research Council grant number $\mathrm{EP} / \mathrm{N} 00275 \mathrm{X} / 1$.

Conflicts of Interest: The authors declare no conflict of interest. The funders had no role in the design of the study; in the collection, analyses, or interpretation of data; in the writing of the manuscript, and in the decision to publish the results.

\section{References}

1. O'Donnell, K.P.; Dierolf, V. (Eds.) Rare-Earth Doped III-Nitrides for Optoelectronic and Spintronic Applications. In Topics in Applied Physics 124; Springer: Dordrecht, The Netherlands, 2010; ISBN 978-90-481-2876-1.

2. O'Donnell, K.P. 2012 UKNC Winter Meeting; University of Bath: Bath, UK. Unpublished talk, 2012.

3. Singh, A.K.; O’Donnell, K.P.; Edwards, P.R.; Lorenz, K.; Kappers, M.J.; Boćkowski, M. Hysteretic photochromic switching of Eu-Mg defects in GaN links the shallow transient and deep ground states of the Mg acceptor. Sci. Rep. 2017, 7, 41982. [CrossRef] [PubMed]

4. Lany, S.; Zunger, A. Dual nature of acceptors in GaN and ZnO: The curious case of the shallow $\mathrm{Mg}_{\mathrm{Ga}}$ deep state. Appl. Phys. Lett. 2010, 96, 142114. [CrossRef]

5. Lyons, J.L.; Janotti, A.; Van de Walle, C.G. Shallow versus deep nature of $\mathrm{Mg}$ acceptors in nitride semiconductors. Phys. Rev. Lett. 2012, 108, 156403. [CrossRef] [PubMed]

6. Singh, A.K.; O’Donnell, K.P.; Edwards, P.R.; Cameron, D.; Lorenz, K.; Kappers, M.J.; Boćkowski, M.; Yamaga, M.; Prakash, R. Luminescence of $\mathrm{Eu}^{3+}$ in $\mathrm{GaN}(\mathrm{Mg}, \mathrm{Eu})$ : Transitions from the ${ }^{5} \mathrm{D}_{1}$ level. Appl. Phys. Lett. 2017, 111, 241105. [CrossRef]

7. Singh, A.K.; O’Donnell, K.P.; Edwards, P.R.; Lorenz, K.; Leach, J.H.; Boćkowski, M. Eu-Mg defects and donor-acceptor pairs in GaN: Photodissociation and the excitation transfer problem. J. Phys. D. 2018, 51, 65106. [CrossRef]

8. O'Donnell, K.P.; Hourahine, B. Rare earth doped III-nitrides for optoelectronics. Eur. Phys. J. Appl. Phys. 2006, 36, 91-103. [CrossRef]

9. O’Donnell, K.P.; Edwards, P.R.; Yamaga, M.; Lorenz, K.; Kappers, M.J.; Boćkowski, M. Crystalfield symmetries of luminescent $\mathrm{Eu}^{3+}$ centers in $\mathrm{GaN}$ : The importance of the ${ }^{5} \mathrm{D}_{0}$ to ${ }^{7} \mathrm{~F}_{1}$ transition. Appl. Phys. Lett. 2016, 108, 022102. [CrossRef]

10. Wang, K.; Martin, R.W.; O’Donnell, K.P.; Katchkanov, V.; Nogales, E. Selectively excited photoluminescence from Eu-implanted GaN. Appl. Phys. Lett. 2005, 87, 112107. [CrossRef]

11. Roqan, I.S.; O’Donnell, K.P.; Martin, R.W.; Edwards, P.R.; Song, S.F.; Vantomme, A.; Lorenz, K.; Alves, E.; Boćkowski, M. Identification of the prime optical center in GaN:Eu ${ }^{3+}$. Phys. Rev. B 2010, 81, 085209. [CrossRef]

12. Mitchell, B.; Poplawsky, J.; Lee, D.; Koizumi, A.; Fujiwara, Y.; Dierolf, V. The role of donor-acceptor pairs in the excitation of Eu-ions in GaN:Eu epitaxial layers. J. Appl. Phys. 2014, 115, 204501. [CrossRef]

13. Lorenz, K.; Miranda, S.M.C.; Alvesa, E.; Roqan, I.S.; O’Donnell, K.P.; Boćkowski, M. High pressure annealing of europium implanted GaN. Proc. SPIE 2012, 2012, 82620C-1. [CrossRef]

14. O’Donnell, K.P.; Edwards, P.R.; Kappers, M.J.; Lorenz, K.; Alves, E.; Boćkowski, M. Europium-doped $\mathrm{GaN}(\mathrm{Mg})$ : Beyond the limits of the light-emitting diode. Phys. Status Solidi C 2014, 11, 662-665. [CrossRef]

15. O'Donnell, K.P. 2015 UKNC Winter Meeting; University of Nottingham: Nottingham, UK. Unpublished talk, 2015.

16. O'Donnell, K.P. 2016 UKNC Winter Meeting; University of Cambridge: Cambridge, UK. Unpublished talk, 2016.

(C) 2018 by the authors. Licensee MDPI, Basel, Switzerland. This article is an open access article distributed under the terms and conditions of the Creative Commons Attribution (CC BY) license (http://creativecommons.org/licenses/by/4.0/). 